PSICOLOGIA

IBEROAMERICANA
Psicología Iberoamericana ISSN: 1405-0943

revista.psicologia@ibero.mx

Universidad Iberoamericana, Ciudad de México México

\title{
Validación de escala para medir las creencias sobre el consumo de alcohol en adolescentes escolarizados
}

Higareda Sánchez, Jesús Javier; Rivera Aragón, Sofía; Reidl Martínez, Lucy María; Flores Galaz, Mirta Margarita; Romero Palencia, Angélica

Validación de escala para medir las creencias sobre el consumo de alcohol en adolescentes escolarizados

Psicología Iberoamericana, vol. 28, núm. 2, 2020

Universidad Iberoamericana, Ciudad de México, México

Disponible en: http://www.redalyc.org/articulo.oa?id=133964928006

Esta obra está bajo una Licencia Creative Commons Atribución 4.0 Internacional.

Esta obra está bajo una Licencia Creative Commons Atribución 4.0 Internacional.

\section{(c) (1)}

Esta obra está bajo una Licencia Creative Commons Atribución 4.0 Internacional. 


\section{Validación de escala para medir las creencias sobre el consumo de alcohol en adolescentes escolarizados}

Psicología Iberoamericana, vol. 28, núm. 2,2020

Universidad Iberoamericana, Ciudad de México, México

Recepción: 01 Junio 2020 Aprobación: 14 Septiembre 2020

Redalyc: http://www.redalyc.org/ articulo.oa?id=133964928006
Scale validation to measure beliefs about alcohol consumption in teenage school students

Jesús Javier Higareda Sánchez javier.higareda88@gmail.com Universidad Nacional Autónoma de México, México

http://orcid.org/0000-0002-9240-0815 Sofía Rivera Aragón Universidad Nacional Autónoma de México, México

http://orcid.org/0000-0003-1170-2430

Lucy María Reidl Martínez

Universidad Nacional Autónoma de México, México

http://orcid.org/0000-0003-4163-2293

Mirta Margarita Flores Galaz

Universidad Autónoma de Yucatán, México

http://orcid.org/0000-0002-9971-8404

Angélica Romero Palencia

Universidad Autónoma del Estado de Hidalgo, México

D http://orcid.org/0000-0003-0933-6937

Resumen: Se propuso diseñar una escala que evaluara las creencias sobre el consumo de alcohol en adolescentes mexicanos escolarizados entre 11 y 16 años, a los cuales se accedió por medio de una invitación. La medida creada tuvo como base la Teoría de la Acción Planeada, la cual explica diversas problemáticas de salud. Se eligieron de manera no probabilística tres muestras de colegios de educación básica del turno matutino del estado de Hidalgo, México. Se llevaron a cabo los pilotos con estas muestras: uno para un estudio cualitativo a través de un análisis de contenido por categorías $(.=219)$, otro para un análisis factorial exploratorio con análisis paralelo $(=906)$ y uno para un análisis factorial confirmatorio por máxima verosimilitud y un análisis de invarianza por sexo (.=612). Los resultados indican la presencia de creencias positivas y negativas de los motivos de consumo en esta población, tanto el AFE y el AFC identificaron dos creencias positivas: placer-hedonismo y aceptación social y una creencia negativa: daño-riesgo. La discusión gira en torno a las creencias conductuales (actitudes) positivas y negativas y en la trasmisión cultural que realizan padres y pares. La limitación es el tipo de muestra ya que solo se trabajó con adolescentes escolarizados, se propone incluir adolescentes que no estudien para continuar con la línea de investigación.

Palabras clave: psicometría, creencias sobre el consumo, cultura, desarrollo humano, adolescencia.

Abstract: This article aims to design a scale to assess beliefs about alcohol consumption in Mexican adolescent students between the ages of 11 and 16-years old, who were invited to be part of the study. The measure created was based on the Theory of Planned Action, which explains various health problems. Three samples from primary schools from the morning shift in the state of Hidalgo, Mexico were chosen using non-probabilistic sampling. The pilots were carried out using these samples: one for a qualitative study through a content analysis by categories $(n=219)$, another for an exploratory factor analysis with parallel analysis $(n=906)$ and one for a factor analysis, confirmatory by maximum likelihood and 
an invariance analysis by sex $(n=612)$. The results indicate the presence of positive and negative beliefs about the reasons for consumption in this population, both the AFE and the AFC identified two positive beliefs: pleasure-hedonism and social acceptance and a negative belief: harm-risk. The discussion revolves around positive and negative behavioral beliefs (attitudes) and the cultural transmission carried out by parents and peers. The type of sample was a limitation of the study since only adolescent students were studied and it would be useful to include adolescents who do not study to continue with this line of research.

Keywords: psychometry, beliefs of consumption, culture, human development, adolescence.

\section{Introducción}

El Consumo de Alcohol (CA) se refiere a la ingesta de etanol encontrado en bebidas alcohólicas (Hammer et al., 2018). La OPS (Organización Panamericana de la Salud, 2015) menciona que los adolescentes en las Américas[2] consumieron su primera bebida alcohólica antes de los 15 años, así mismo, la Encuesta Mundial de Salud[3] realizada a escolares (WHO, 2013), que incluyó a 23 países de América, señaló que los estudiantes de entre 13 y 17 años consumieron por lo menos una bebida alcohólica estándar en su vida (12 g. de alcohol); los hombres consumieron más en comparación con las mujeres y el $20 \%$ bebe hasta la intoxicación.

En México, de acuerdo con la Encuesta Nacional de Consumo de Drogas Alcohol y Tabaco, el consumo de alcohol en adolescentes tuvo aumentos significativos en los rubros: consumo excesivo (cinco o más copas en el último mes), de $4.3 \%$ a $8.3 \%$, consumo consuetudinario (consumo de cinco o más copas a la semana) del $1 \%$ a $4.1 \%$ y consumo diario (una copa al menos al día) de $.04 \%$ a $2.5 \%$. De igual forma, se presentaron aumentos significativos en el CA por sexo en todos los rubros antes mencionados con respecto a la medición de 2011 (VillatoroVelázquez et al., 2017) . La Encuesta Nacional de Consumo de Drogas en Escolares señaló que cerca del 35\% de estudiantes de secundaria y bachillerato habían consumido alcohol; la bebida preferida fue cerveza y que $15.4 \%$ presentaron consumo excesivo (Secretaría de Salud, 2015).

Una de las alteraciones debidas al consumo excesivo de alcohol en adolescentes, son las asociadas con el desarrollo neurológico, el etanol altera el desarrollo de áreas específicas como el córtex frontal, encargado del procesamiento de la información y la memoria (Spear, 2002). Además, con el paso del tiempo tiene relación con otros comportamientos como el consumo de marihuana y tabaco (Oliva-Delgado et al., 2008) y las relaciones coitales sin protección (Pechorro et al., 2018). Al mismo tiempo, beber en exceso antes de los 15 años tiene consecuencias como: mayor probabilidad de presentar dependencia al alcohol, sufrir un accidente automovilístico, estar envuelto en una pelea y presentar consumo de riesgo en la adultez (Ringwalt et al., 2020; Wilsnack et al., 2018). Así, cualquier tipo de consumo en este grupo de la población se considera de riesgo debido a su relación con consecuencias perjudiciales (Inter-American Observatory on Drugs, 2019). 
Las y los adolescentes tienen cambios físicos, psicológicos y sociales durante esta etapa, también pueden presentar riesgos, vulnerabilidades y búsqueda de sensaciones que generan creencias sobre el CA como: mejora en el desempeño social, expresión de sentimientos, aceptación del grupo de referencia, alegría, ocio, relajación y estatus, que en su conjunto, determinan la actitud del consumo de riesgo (Martínez-Cardona et al., 2019; Patrick et al., 2010; Peltzer et al., 2017; Settles et al., 2014; Shell et al., 2010). Por otro lado, creencias negativas como: tener malas experiencias con la sedación, tener conflictos con la familia por consumir y meterse en problemas, son identificadas como factores de protección (Maggs et al., 2015). En general los preadolescentes muestran mayores creencias negativas hacia el CA, sin embargo, conforme crecen desarrollan creencias positivas incluso si el consumo no se ha iniciado (Goldman et al., 2006).

Las creencias pueden ser positivas o negativas y están vinculadas con las consecuencias de consumir (Goldman et al., 1999, 2006; Randolph et al., 2013). En este sentido la Teoría de la Acción Planeada (TAP) explica cómo las creencias anteceden la intención de realizar una conducta, uno de sus principales elementos son las actitudes, las cuales tienen relación con la conducta de beber (Guzmán-Facundo et al., 2018).

En la TAP (Ajzen \& Driver, 1992) las creencias sobre realizar una conducta tienen implicaciones importantes en la intención de realizarla, ya que, si una persona tiene una serie de creencias positivas sobre los posibles resultados de llevarla a cabo, tendrá una actitud positiva, y por el contrario las creencias negativas generarán una actitud negativa (Montaño \& Kasprzyk, 2015). En esta teoría existen tres tipos de creencias: conductuales, normativas y de control. Las creencias conductuales indican la actitud que las personas tendrán antes de realizar una conducta, con base en el atributo o algún resultado que puede ser positivo o negativo. Las creencias normativas indican qué tanto los referentes importantes de cada persona como: la familia, los amigos o la pareja, aprueban o desaprueban el desarrollo de la conducta, este elemento hace referencia al contexto sociocultural en el que la persona se desenvuelve, y consiste en la presión social ejercida para consumir alcohol (López-Cisneros et al., 2013) y las creencias de control indican qué tantos recursos, requisitos o experiencias previas tiene una persona para realizar la conducta.

Estudios con adolescentes indican que las principales creencias sobre el CA son las asociadas con: diversión y relajación, poder conocer gente nueva, olvidar problemas, conseguir pareja, divertirse con amigos, sentirse integrado y tener estatus, estas creencias están vinculadas con los aprendizajes culturales y sociales (Martínez-Cardona et al., 2019), con los amigos y la familia y están relacionadas con las actitudes positivas ante el consumo (Tavares et al., 2019).

Cada cultura indica qué, cómo, cuándo y con quién consumir alcohol, ya que existen ritos y tradiciones familiares y sociales en México (Berruecos, 2013) y en otros contextos de América que guardan similitudes con la cultura mexicana como, por ejemplo, Colombia 
(Castaño Pérez et al., 2014). Estos valores y creencias culturales se transmiten por medio de la familia (padres), por medio de pares y por medio de otros adultos e instituciones. De igual forma las conductas apropiadas, los valores, las creencias y las normas del grupo cultural se transmiten por medio de la socialización. Los padres refuerzan o castigan conductas deseables a la cultura. Los pares socializan por medio de la interacción diaria y las instituciones formales como la escuela o la iglesia contribuyen en este proceso de trasmisión (Berry, 1992; Berry et al., 2011). En este sentido los adolescentes adquieren las normas, creencias y valores que son interiorizadas por medio de la interacción con el grupo y tienen la función de construir la identidad personal (Woolfolk, 2010). Por lo tanto, consumir sustancias forma parte de la cultura juvenil e involucra crear un medio para la socialización con otros adolescentes, donde puedan compartir en un espacio común (Pons \& Buelga, 2011).

Sobre la medición de las creencias sobre el CA se identifica la escala de creencias conductuales en universitarios españoles, cuenta con ítems que evalúan creencias positivas asociadas al consumo (sentirse más confiado, olvidarse de los miedos, mejora en el estado de ánimo) y creencias o consecuencias negativas (verse envuelto en riñas, ser grosero, generar problemas). Esta escala fue creada ad hoc para evaluar el comportamiento planificado y no presenta características psicométricas, además de carecer de un estudio exploratorio previo (Cortés et al., 2011).

De la misma forma se encontró una escala que evalúa el proceso de la teoría de la acción planeada en niños mexicanos, presenta un estudio exploratorio cualitativo previo que usó la técnica de grupos focales con el que se redactaron los reactivos que fueron sometidos a prueba. En la medición de las actitudes se obtuvieron tres factores: Importancia de la creencia (Probar cerveza cuando tenga un problema es); Evaluación de la creencia (Probar cerveza para calmarme cuando estoy enojado es) y Evaluación de la creencia positiva o negativa (Probar cerveza para relajarme es). Obtuvieron un Alpha de .88 y una varianza explicada de $56 \%$, sin embargo, su uso es para población infantil (Hernández Chávez et al., 2017).

De igual forma se ubicó la escala de expectativas sobre el CA la cual identifica expectativas como: mejora en habilidades sociales, disminución del estrés y facilitación social. Cuenta con un porcentaje de varianza explicada de 61\% y un Alpha de Cronbach de .84. La población con quien se validó esta escala fue con población adulta que se encontraba internada en una residencia para adicciones en México y no necesariamente con adolescentes (Templos-Núñez et al., 2013).

Las creencias sobre el CA reflejan parte de la visión sociocultural del consumo debido a que son parte de los elementos subjetivos de la cultura. Se propone la creación de una medida para evaluar este fenómeno basada en 3 estudios piloto, los cuales comienzan con una exploración cualitativa con población adolescente, seguida de la creación de ítems y la extracción de sus elementos psicométricos por medio de un Análisis Factorial Exploratorio (AFE); seguido de un Análisis Factorial 
Confirmatorio (AFC) para conocer los elementos globales de las creencias sobre el CA en adolescentes.

\section{Piloto 1}

Las creencias sobre el CA forman parte de los elementos subjetivos de la cultura las cuales se trasmiten por medio de los principales grupos donde los adolescentes se desarrollan, en este sentido las creencias sobre la iniciación y mantenimiento del CA pueden estar reforzadas desde casa, con amigos o en la comunidad (Berry et al., 2011; Jones, 2016; Matsumoto, 2001; Pons \& Buelga, 2011). Sin embargo, es necesario explorar este fenómeno desde la vivencia misma de los adolescentes que se encuentran inmersos en la cultura mexicana, ya que cada cultura señala los qué, cuándo, dónde y porqué de consumir alcohol (Castaño Pérez et al., 2014).

\section{Método Piloto Uno}

\section{Participantes}

Mediante un muestreo no probabilístico por conveniencia se seleccionó a 219 estudiantes de educación secundaria baja (educación básica de México) con base en la Clasificación Internacional Normalizada de la Educación (UNESCO, 2013), pertenecientes a tres colegios públicos con turno matutino, ubicados en la capital del estado de Hidalgo, México. Con una media de edad de $13.85(D E=1.11)$ con un rango de edad de 11 a 16, los cuales 118 fueron hombres (54\%) y 101 fueron mujeres (46\%). En cuanto al grado que cursaban $101(46.11 \%)$ cursaban el tercer grado, $64(29.22 \%)$ segundo y $54(24.65 \%)$ el primer grado. Finalmente 112 (51.14\%) vivían con ambos padres, 83 (37.89\%) vivían con algún padre, y $24(10.94 \%)$ vivían con los abuelos o algún otro familiar.

\section{Instrumento}

Se elaboró un cuestionario autoaplicado de cuatro preguntas abiertas para explorar las creencias personales (¿qué obtiene una persona de su edad al consumir alcohol?), de la familia (iqué cree su familia sobre el consumo de alcohol en personas de su edad?), la comunidad (¿qué cosas, dichos o consejos ha escuchado sobre consumir alcohol?) y los amigos (¿qué piensan sus amigos sobre el consumo de alcohol en personas de su edad?), además este cuestionario incluía cuatro preguntas sociodemográficas (edad, sexo, dónde vivían y con quién). 


\section{Procedimiento}

Se asistió a las escuelas a presentar el proyecto a los directivos y a los padres de familia de escuelas secundarias. Los padres firmaron el consentimiento de la aplicación, se invitó de manera verbal a los estudiantes a participar, señalando que su participación era voluntaria, anónima y que sus respuestas serían de uso estadístico, ellos mismos firmaron el asentimiento. Los datos fueron recabados por el investigador principal y fueron supervisados por las autoridades de las escuelas. Después de la aplicación se agradeció a los participantes y se brindaron datos sobre instituciones de salud mental gratuitos. Los datos fueron recabados entre los meses de noviembre 2016 y febrero 2017.

\section{Análisis de Datos}

Las respuestas fueron transcritas y analizadas por medio del programa estadístico Excel. Se realizó un análisis de contenido por medio de categorías emergentes, este análisis permite aglutinar las respuestas de los participantes en categorías de familias conceptuales creadas (Krippendorff, 2018). Las respuestas de los participantes fueron asignadas a una serie de categorías que comparten características semánticas, las cuales son definidas con base en la teoría de la acción planeada (actitudes), contabilizadas y presentadas en orden de aparición, en este sentido la categoría con más aparición al inicio y al final la que menos se presentó, finalmente se complementan las definiciones con ejemplos de transcripciones.

\section{Resultados}

Las categorías emergentes señalan creencias asociadas con consecuencias negativas como: es una actividad peligrosa o que hace daño. Así como otras respuestas como el cambio en el humor y socialización, por ejemplo: que te brinda placer, bienestar, te relaja, te ayuda a ser aceptado por los amigos y que es algo normal para las personas, para presentar los resultados se describe la categoría emergente, con indicadores del discurso y el número de veces de la aparición de los mismos (ver Tabla 1). 


\section{Tabla 1}

Análisis de contenido de las creencias sobre el CA en adolescentes $(\mathrm{N}=219)$

\section{Tabla 1}

Análisis de contenido de las creencias sobre el CA en adolescentes $(N=219)$

\begin{tabular}{|c|c|c|c|}
\hline Categoría & Definición & Ejemplo de indicadores & $\begin{array}{l}\text { Aparición de } \\
\text { los } \\
\text { indicadores }\end{array}$ \\
\hline Daño-riesgo & $\begin{array}{l}\text { Creencias negativas } \\
\text { sobre que el alcohol } \\
\text { tiene repercusiones } \\
\text { físicas, psicológicas y } \\
\text { sociales en la persona } \\
\text { que consume alcohol }\end{array}$ & $\begin{array}{l}\text { "es malo", "enfermedad", } \\
\text { "hacerse daño", "daños a la } \\
\text { salud" }\end{array}$ & 392 \\
\hline $\begin{array}{l}\text { Aceptación } \\
\text { grupal }\end{array}$ & $\begin{array}{l}\text { Creencias positivas } \\
\text { sobre que el consumo } \\
\text { de alcohol es necesario } \\
\text { para ser aceptado por el } \\
\text { grupo de amigos o el } \\
\text { grupo de referencia }\end{array}$ & $\begin{array}{l}\text { "para ser aceptado por el } \\
\text { grupo", "para ser aceptado en } \\
\text { el grupo social", "los amigos te } \\
\text { invitan", "los amigos te invitan } \\
\text { a fiestas" }\end{array}$ & 174 \\
\hline $\begin{array}{l}\text { Placer- } \\
\text { bienestar }\end{array}$ & $\begin{array}{l}\text { Creencias positivas } \\
\text { sobre que el consumo } \\
\text { de alcohol tiene efectos } \\
\text { relajantes o de } \\
\text { enfrentamiento a } \\
\text { situaciones estresantes }\end{array}$ & $\begin{array}{l}\text { "que son ricas y te } \\
\text { desestresan", "sirve para } \\
\text { olvidar sus problemas", } \\
\text { "olvidar sus problemas", } \\
\text { "disfrutar la vida", "para } \\
\text { distraerse y } \\
\text { divertirse con sus } \\
\text { amigos", "para sentirse bien" }\end{array}$ & 74 \\
\hline $\begin{array}{l}\text { Normalidad/ } \\
\text { aprobación }\end{array}$ & $\begin{array}{l}\text { Creencias sobre que el } \\
\text { consumo de alcohol en } \\
\text { adolescentes es algo } \\
\text { normal o común y que la } \\
\text { familia y el grupo lo } \\
\text { aprueba. }\end{array}$ & $\begin{array}{l}\text { "dejando que beban } \\
\text { alcohol", "prefiero que tomes } \\
\text { aquí en la casa", "algo } \\
\text { normal", "nada de malo", } \\
\text { "necesario para tener } \\
\text { ambiente", "que sí puede } \\
\text { tomar para ver si le gusta o } \\
\text { no", "que tarde o temprano lo } \\
\text { van a tener que probar y que } \\
\text { es mejor } \\
\text { enfrente de ellos y no a sus } \\
\text { espaldas" }\end{array}$ & 65 \\
\hline Estatus & $\begin{array}{l}\text { Creencias positivas } \\
\text { sobre que consumir } \\
\text { alcohol brinda una } \\
\text { posición superior dentro } \\
\text { del grupo de pares } \\
\text { donde se desenvuelve el } \\
\text { adolescente. }\end{array}$ & $\begin{array}{l}\text { "ya tienen edad para hacer lo } \\
\text { que quieras", "es de } \\
\text { valientes", "es de grandes", } \\
\text { "para dejar de ser niño" }\end{array}$ & 21 \\
\hline
\end{tabular}




\section{Discusión Piloto Uno}

Los resultados de este piloto muestran que las creencias son un elemento importante para desarrollar el consumo. Las actitudes de la familia, los pares y el contexto, tienen una fuerte influencia en la formación de creencias y desarrollo de conductas de beber en adolescentes ya que las relaciones proximales desarrollan la percepción de riesgo o beneficio (Goldman et al., 1999, 2006; Randolph et al., 2013; Woolfolk, 2010).

Las creencias de daño-riesgo son socializadas por la educación formal y la prevención del uso de drogas, de igual forma son usadas por adultos que desean postergar el consumo en sus hijos, ya que la familia y otras estructuras transmiten esta información (Chan et al., 2017). Por ejemplo, en México, cerca de 2.5 millones de personas fueron atendidas de manera preventiva, los cuales un millón y medio fueron niños y adolescentes escolarizados, que equivalen al 68\% (CIJ, 2020), lo anterior indica que la información sobre las consecuencias del CA llega a estas poblaciones, sin embargo, los datos de consumo de riesgo y consuetudinario aumentaron con respecto a los años 2011 y 2017 (Villatoro-Velázquez et al., 2017). Es posible que este aumento en el CA se deba a la socialización y la exacerbación de las consecuencias positivas sobre el consumo de alcohol en población adulta y otros pares.

Los amigos que tienen creencias negativas son un factor de protección ante este problema (Chan et al., 2017). La TAP señala que la conducta individual estará influenciada por los factores ambientales y personales, así una persona desarrollará la conducta con base en si sus expectativas de resultado son positivas o negativas (Ajzen \& Driver, 1992). Así, si la familia o los pares tienen creencias negativas, menor será la probabilidad de que el CA en adolescentes se presente (Randolph et al., 2013). Sin embargo, el consumo del grupo de pares puede ser una de las actividades principales a desarrollar con el objetivo de ser aceptado (López-Cisneros et al., 2013), lo que hace que beber sea una actividad deseable socialmente. Con base en lo anterior se crearon ítems para someter a prueba la primera versión de la escala de creencias sobre el CA en adolescentes.

\section{Piloto Dos}

Las escalas que miden las creencias sobre el CA están centradas en las consecuencias positivas y negativas que experimentan las personas cuando consumen. Sin embargo, algunas no indican si existen estudios exploratorios previos con población meta. Cada civilización o grupo tiene sus propias creencias sobre el alcohol, ya que se trata de un artefacto cultural que cuenta con una muy importante carga simbólica con sus propias normas y creencias que indican su uso (CIJ, 2020). 


\section{Método Piloto Dos}

\section{Participantes}

Participaron 906 estudiantes de educación secundaria baja (UNESCO, 2013) de tres colegios públicos de turno matutino, ubicados en la ciudad de Pachuca Hidalgo, México; seleccionados por medio de un muestreo no probabilístico por conveniencia con una media de edad de 13.37 ( $\mathrm{DE}=1.09)$ y un rango de 11 a 16 , los cuales $458(50.6 \%)$ fueron hombres; $360(39.7 \%)$ cursaban el primer grado y 540 (59.6\%) vivían con ambos padres.

\section{Instrumentos}

Escala de Creencias Sobre el Consumo de Alcohol para Adolescentes la cual se elaboró con base en el piloto uno. Tuvo 50 ítems con opción de respuesta tipo Likert de cuatro puntos ( $1=$ nunca a $4=$ siempre) que evaluaban las creencias sobre el consumo de alcohol en adolescentes.

\section{Procedimiento}

Los padres firmaron el consentimiento de la aplicación, se invitó de manera verbal a los estudiantes a participar en el estudio, señalando que su participación era voluntaria y anónima y que sus respuestas serían de uso estadístico, ellos mismos firmaron el asentimiento. En este caso la aplicación duró aproximadamente 30 minutos. Se supervisaron las aplicaciones por parte del investigador en caso de existir dudas. Los cuestionarios fueron aplicados en las diferentes escuelas entre los meses de diciembre de 2017 y junio de 2018.

\section{Análisis de Datos}

Se realizó una validación culturalmente relevante (Reyes- Lagunes \& García y Barragán, 2008) que consta del análisis de frecuencias, discriminación de reactivos por grupos extremos y la correlación entre cada ítem con la escala total.

\section{Discriminación de Ítems}

Por medio del IBM SPSS versión 22 se realizaron análisis de frecuencias de los reactivos para conocer si existieron respuestas en cada una de las opciones de la escala. Se realizaron análisis de sesgo para conocer la distribución de los ítems. Después se compararon el cuartil primero con el cuartil cuarto para saber si los grupos extremos discriminaban entre sí por medio de la prueba t de Student. Finalmente se realizaron correlaciones de Pearson entre los reactivos y el puntaje de la escala total. 


\section{Análisis Factorial Exploratorio (AFE)}

Por medio del software Factor (Ferrando \& Lorenzo-Seva, 2007) se realizó el AFE, para obtener el número de dimensiones, se utilizó el análisis paralelo (Timmerman \& Lorenzo-Seva, 2011) el cual analiza las correlaciones policóricas y tetracóricas de datos nominales u ordinales (Choi et al., 2011). La rotación utilizada fue Promax (oblicua) debido a la relación entre los ítems (Martínez \& Sepúlveda, 2012) y el método de extracción fue por medio de Cuadrados mínimos no ponderados (Unweighted Least Squares) debido a que los datos tuvieron una distribución no normal (Ximénez-Gómez \& García, 2005). Se eliminaron aquellos reactivos con comunalidades por debajo de $.30 \mathrm{y}$ se estableció el punto de corte de .40 para conservar ítems dentro de los factores generados con autovalores por arriba de uno. También se eliminaron los reactivos que cargaban en más de un factor por arriba de .40 (Tabachnick \& Fidell, 2007).

\section{Análisis de Consistencia Interna}

Por medio de un análisis de alpha de Cronbach por factor generado y por la escala total, se obtuvo la confiablidad (Nunnally \& Bernstein, 1995).

\section{Resultados}

Se obtuvo una escala de 25 reactivos que se agruparon en tres factores: Daño-riesgo, Aceptación social y Hedonismo-placer, los cuales explicaron el $68.27 \%$ de la varianza (validez de constructo) y una consistencia interna total de $\alpha=.86$. Se decidió mantener el reactivo: beber alcohol ayuda a superar las penas, en el factor dos, debido a que teóricamente fue relevante. En la Tabla 2 se observan las cargas factoriales, el número de ítems y la varianza explicada por factor y en la Tabla 3 las definiciones de los factores obtenidos. 
Tabla 2

Cargas factoriales del AFE de la Escala de Creencias Sobre el Consumo de Alcohol en Adolescentes

Tabla 2

Cargas factoriales del AFE de la Escala de Creencias Sobre el Consumo de Alcohol en Adolescentes

\begin{tabular}{|c|c|c|c|c|}
\hline İtem & Factor 1 & Factor 2 & Factor 3 & Total \\
\hline Es peligroso & .73 & -.03 & .08 & \\
\hline Es grave & .73 & -.02 & .07 & \\
\hline Mata & .82 & -.02 & .11 & \\
\hline Afecta la salud & .93 & -.08 & .02 & \\
\hline Es un vicio & .79 & .01 & .07 & \\
\hline Te enferma & .79 & .13 & -.14 & \\
\hline Es malo & .85 & .04 & -.18 & \\
\hline Te da personalidad & -.03 & .60 & .22 & \\
\hline Te ayuda a hacer amigos & .01 & .71 & .11 & \\
\hline Es de valientes & -.05 & .77 & .04 & \\
\hline Ayuda a ser aceptado por amigos & .01 & .83 & .01 & \\
\hline Te hace más hombre o más mujer & -.01 & .82 & .01 & \\
\hline Ayuda a formar parte del grupo & .07 & .94 & $\begin{array}{l}-.12 \\
-07\end{array}$ & \\
\hline $\begin{array}{l}\text { Es parte de tener amigos } \\
\text { Ayuda a superar penas }\end{array}$ & $\begin{array}{l}.01 \\
.07\end{array}$ & $\begin{array}{l}.87 \\
.36\end{array}$ & $\begin{array}{r}-.07 \\
.31\end{array}$ & \\
\hline Ayuda a olvidar amores & .07 & .48 & .18 & \\
\hline Te hace fuerte & -.05 & .51 & .25 & \\
\hline Está bien & -.03 & -.09 & .85 & \\
\hline Es rico & .06 & -.16 & .93 & \\
\hline Te da placer & .04 & .17 & .69 & \\
\hline Es divertido & -.02 & .15 & .72 & \\
\hline Es bueno & -.14 & .02 & .82 & \\
\hline Es chido & -.01 & .16 & .72 & \\
\hline Es un gusto & .09 & .12 & .63 & \\
\hline Se siente bien & .05 & .05 & .78 & \\
\hline Numero de ítems & 7 & 10 & 8 & 25 \\
\hline Varianza explicada & $41 \%$ & $20 \%$ & $7 \%$ & $68 \%$ \\
\hline Alfa de Cronbach & .88 & .84 & .89 & .86 \\
\hline
\end{tabular}


Tabla 3

Definición de los factores de la Escala de Creencias Sobre el Consumo de Alcohol en Adolescentes Tabla 3

Definición de los factores de la Escala de Creencias Sobre el Consumo de Alcohol en Adolescentes

Daño-riesgo Se refiere a las creencias sobre el consumo de alcohol asociadas a las consecuencias negativas en salud y sociales.

Aceptación Son las creencias sobre el consumo de alcohol relacionadas con social las consecuencias sociales y personales positivas que ocurren al consumir alcohol.

Hedonismo- Se refiere a las creencias sobre el consumo de alcohol placer relacionadas con experimentar cambios físicos y psicológicos asociados con bienestar, relajación y afecto positivo.

\section{Discusión Piloto Dos}

El AFE indicó que el factor Daño-riesgo, se mantuvo, bajo la perspectiva del análisis de costos y beneficios del CA, los adolescentes tomarán decisiones sobre beber con base en la evaluación razonada de las consecuencias (Gerrard et al., 1996). Esto se explica también desde la teoría de las expectativas la cual indica que las personas presentan CA debido a que tienen creencias sobre lo que ocurrirá en el futuro y que pueden ser creadas con base en la observación del entorno (Jones et al., 2001) si estas expectativas son negativas como que "es malo", "enferma" o "mata" el consumo será menor o nulo (Rodriguez et al., 2019).

En otros estudios este factor es un factor protector en adolescentes (Hodder et al., 2018), ya que, tener creencias sobre el daño a la salud y otras consecuencias se asociaron con la disminución en el CA en dos mediciones entre 2011 y 2016 con adolescentes de Australia, los autores puntualizan que este tipo de creencias en conjunto con otros factores protectores personales, sociales y ambientales contribuyeron a la disminución del CA en esta población.

Por el contrario tener creencias positivas sobre las consecuencias de consumir, tendrán un efecto en el consumo de adolescentes (Woolfolk, 2010) estos factores positivos también han aparecido en escalas como la de creencias conductuales (Ahumada-Cortez et al., 2017) la cual contiene ítems como: mejora en el estado de ánimo, sentirse confiado, enfrentar los miedos bebiendo, esta escala junto con otras escalas como la de 
expectativas del consumo (Templos-Núñez et al., 2013) presentan la configuración de valencias positivas y negativas sobre las creencias del consumo.

\section{Método Piloto Tres}

\section{Participantes}

Participaron 612 estudiantes con las mismas características de los pilotos previos de tres colegios públicos de turno matutino de la ciudad de Pachuca, Hidalgo, seleccionados por medio de un muestreo no probabilístico por conveniencia con una media de edad de 13.62 $(\mathrm{DE}=.88)$ con un rango de 11 a 16 , de los cuales 318 fueron mujeres (52\%), 324 cursaban el tercer año (52.9\%) y 398 (65\%) vivían con ambos padres.

\section{Instrumentos}

Escala de Creencias Sobre el Consumo de Alcohol en Adolescentes (Piloto 2). Con una escala de respuesta tipo Likert de cuatro puntos ( $1=$ nunca a $4=$ siempre $)$. Compuesto por tres factores: Daño-riesgo, Aceptación social y Hedonismo-placer.

\section{Procedimiento}

Se respetaron los criterios de anonimato, confidencialidad y participación voluntaria, padres y alumnos firmaron el consentimiento y asentimiento informado. Los cuestionarios fueron aplicados en escuelas de la capital del estado de Hidalgo con turno matutino, entre los meses de enero 2019 y agosto de 2019. Los datos se capturaron en el programa SPSS versión 21 y se analizaron con el programa SPSS AMOS versión 24.

\section{Análisis de Datos}

Se propuso un Análisis Factorial Confirmatorio (AFC) con el método de Máxima Verosimilitud, se consideraron los índices $\chi 2 / \mathrm{gl}$ con valores esperados menores a 3, GFI (Goodness of Fit Index) con valores mayores a .90 como aceptables; SRMR (Standardized Root of Mean Squared Residual) menores a .08 y RMSEA (Root Mean Squaer Error of Approximation) con valores de .08 o menores como aceptables. Finalmente se llevó a cabo un análisis de invarianza por sexo. 


\section{Resultados}

\section{Modelo General}

Se eliminó el ítem 49 (beber alcohol te hace fuerte) debido a que su carga factorial se encontraba por debajo de .40. Se covariaron tres errores, uno en cada factor. El modelo ajustó adecuadamente $(\chi 2=681.737$, $\mathrm{gl}=246 ; \quad \mathrm{CMIN} / \mathrm{DF}=2.771 ; \mathrm{GFI}=.913 ; \mathrm{CFI}=.935 ; \mathrm{SRMS}=.056 \mathrm{y}$ RMSEA=.054). Los índices GFI y RMSEA son aceptables de acuerdo con Ferrando-Piera y Anguiano-Carrasco (2010) y los índices $\chi 2 / g l$ y SRMR indican un buen ajuste del modelo (Abad, 2011). En la Figura 1 se muestra la estructura factorial de la escala.

\section{Figura 1}

Estructura factorial de la escala de Creencias sobre el consumo de alcohol en adolescentes $(N=612)$

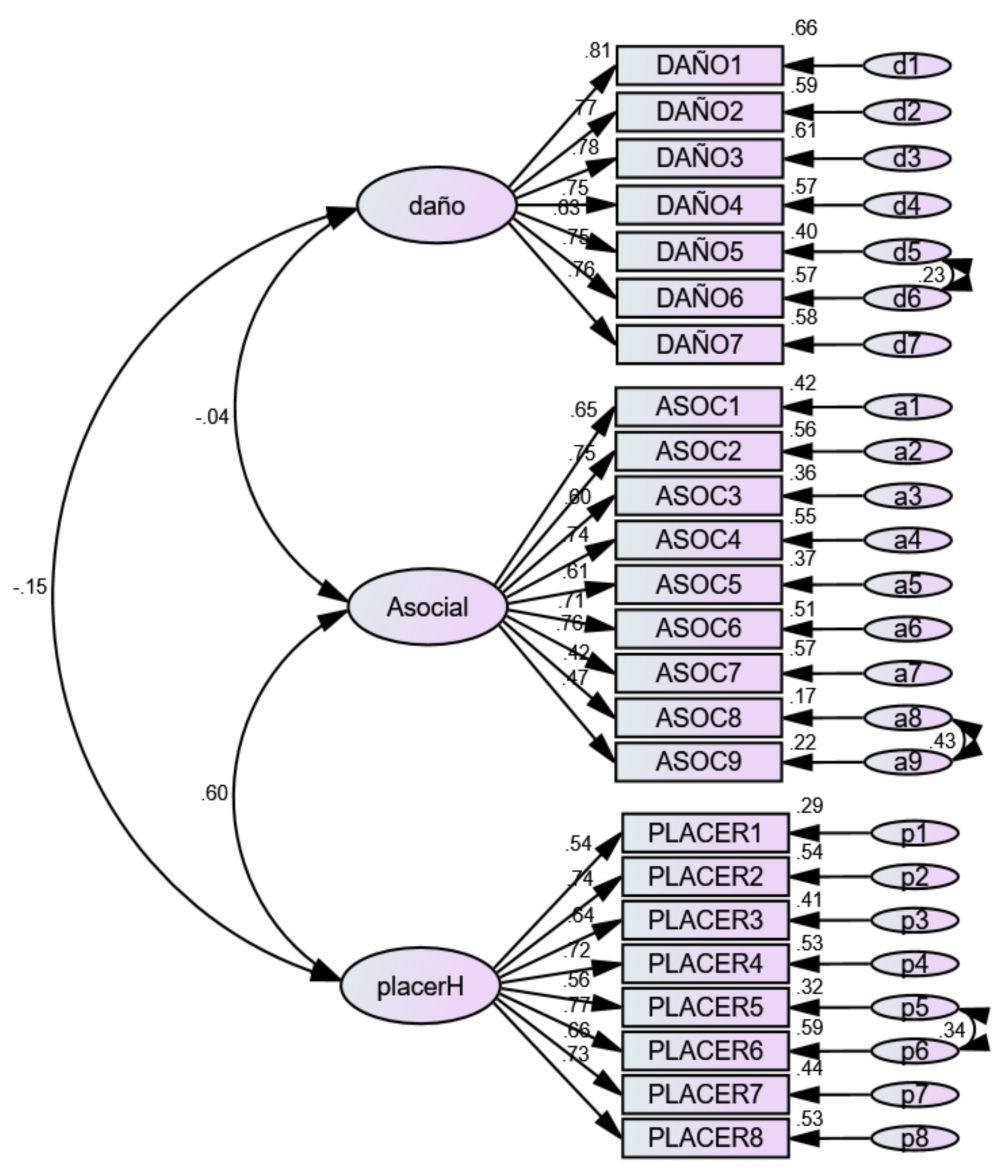

Figura 1

Estructura factorial de la escala de Creencias sobre el consumo de alcohol en adolescentes $(\mathrm{N}=612)$ 


\section{Análisis de Invarianza por Sexo}

Para este análisis se dividió la muestra por sexos (hombres=294 y mujeres=318). Se sometieron a prueba dos AFC, uno para cada sexo y presentaron un ajuste adecuado por grupo. Dos AFC adicionales fueron sometidos a prueba. El primero llamado modelo configurado, el cual evalúa si el número de factores y sus cargas son equivalentes entre grupos (Brown, 2006). Este modelo mostró un adecuado ajuste, lo que indica equivalencia entre grupos. El último AFC evaluó la igualdad en las cargas factoriales y covarianzas de los errores, lo que indica la especificación de restricciones de igualdad para todas las cargas de factores libremente estimadas en ambos modelos (Byrne, 2012), el análisis indicó un ajuste adecuado (ver Tabla 4).

Tabla 4

Resultados del análisis de invarianza del AFC de la Escala de Creencias Sobre el Consumo de Alcohol en Adolescentes por sexo

\section{Tabla 4}

Resultados del análisis de invarianza del AFC de la Escala de Creencias Sobre el Consumo de Alcohol en Adolescentes por sexo

\begin{tabular}{|c|c|c|c|c|c|c|c|c|}
\hline & $x^{2}$ & $d f$ & $\begin{array}{l}x^{2} \\
\text { diff }\end{array}$ & $\Delta d f$ & CFI & RMSEA & $\begin{array}{c}\text { RMSEA } \\
\text { C/90 }\end{array}$ & SRMR \\
\hline \multicolumn{9}{|l|}{$\begin{array}{l}\text { Grupos } \\
\text { únicos }\end{array}$} \\
\hline $\begin{array}{l}\text { Hombres } \\
(n=294)\end{array}$ & $568.909^{*}$ & 246 & & & 0.90 & 0.07 & $.061-.076$ & 0.06 \\
\hline $\begin{array}{l}\text { Mujeres } \\
(n=318)\end{array}$ & $458.599^{*}$ & 246 & & & 0.92 & 0.05 & $.045-.060$ & 0.06 \\
\hline $\begin{array}{l}\text { Modelo } \\
\text { configurado }\end{array}$ & $1027.508^{*}$ & 492 & & & 0.91 & 0.04 & $.039-.047$ & 0.06 \\
\hline $\begin{array}{l}\text { Medición } \\
\text { del modelo } \\
\text { de } \\
\text { invarianza }\end{array}$ & $1112.524^{*}$ & 513 & 85.01 & 21 & 0.90 & 0.04 & $.041-.048$. & 0.07 \\
\hline
\end{tabular}

\section{Discusión Piloto Tres}

La teoría de la acción planeada señala que las creencias o motivaciones sobre realizar una conducta son importantes para realizarla (Ajzen \& Driver, 1992). En esta medida creada se identifican las actitudes sobre realizar la conducta de beber alcohol en adolescentes entre 11 y 16 años de México. Las actitudes pueden ser positivas o negativas y reflejan la evaluación de los factores individuales sobre la conducta en cuestión (Ajzen, 2002) y en estudios con adolescentes este elemento es el que 
explica con mayor fuerza la intención de consumir en ambos sexos (Golpe et al., 2017).

Sobre el AFC se observa que se mantuvieron los reactivos propuestos previamente, sin embargo, se covariaron dos errores (Beber alcohol es grave y Beber alcohol mata), en este sentido ambos ítems señalan consecuencias perjudiciales graves. Teóricamente ambos son relevantes debido a que son las consecuencias más letales, además de ser las consecuencias a largo plazo que los adolescentes identifican (Musitu Ochoa, 2014), sin embargo, al ser identificadas como consecuencias a largo plazo no tienen un efecto protector, sino son identificadas como una serie de discursos preventivos sanitarios que no tienen impacto en la realidad (Pons \& Buelga, 2011).

Los elementos positivos del CA en esta población están vinculados con actividades a realizar en entornos propios de la juventud, donde el consumo entre pares surge como una actividad en espacios comunes donde puedan interactuar (Higareda et al., 2018). Sobre la aceptación grupal se sabe que en esta etapa los adolescentes pueden experimentar una serie de presiones acerca de iniciarse en el consumo y esta presión puede ser directa en forma de invitaciones y otra indirecta en forma de creencias normativas acerca de lo que debe de hacer un adolescente, en algunas ocasiones el hecho de que el adolescente no se inicie en la dinámica del grupo, puede tener como consecuencia la exclusión social (Cortés et al., 2011; Higareda et al., 2018).

De igual forma, en esta etapa el adolescente suele consumir para desarrollar su identidad, la cual tiene un fuerte componente social (Bustos-Gamiño et al., 2019; Rodriguez et al., 2019). En situaciones sociales el alcohol puede fungir como un facilitador social y para que realicen actividades que no harían en otras situaciones. En los jóvenes se vincula con el consumo de los amigos y en actitudes favorables sobre el CA las cuales tienen relación positiva con la iniciación del consumo (Rodriguez et al., 2019). De igual manera se asocia con el desarrollo de la identidad individual y grupal de los adolescentes, como también lo hacen el gusto musical, el vestuario y la inversión del tiempo libre. Estos elementos podrían estar relacionados con que los adolescentes generen actitudes positivas sobre el alcohol (Ahumada-Cortez et al., 2017) en espacios donde pueden disfrutar del estatus de hacer cosas que sólo los adultos realizan, como beber, tener conductas de riesgo, iniciar la vida sexual, mostrar su hombría y fortaleza etc. (Musitu Ochoa, 2014), estas conductas tienen una base social de imitación de los ambientes adultos, que son reforzadas por el grupo de pares que simbolizan madurez.

El CA es una actividad desarrollada en México, una actividad que involucra a la familia, al disfrute de las reuniones, al placer de estar con amigos y familiares. Este modelo adulto resulta importante ya que los adolescentes reproducen estas creencias y conductas en sus grupos, donde beber es visto como una actividad normal, o socialmente aceptada, incluso algunos adolescentes mencionan que son los adultos quienes señalan las consecuencias negativas, aunque en casa son los mismos adultos quienes desarrollan esta actividad, contradiciendo lo que los padres quieren y 
lo que hacen, estas contradicciones contribuyen a la legitimización o normalización del consumo (Higareda et al., 2018), lo que da lugar al consumo en casa donde los adultos supervisan lo que los menores beben (Donovan et al., 2009).

El AFC indicó que los reactivos que explicaban el factor aceptación social miden la presencia de creencias positivas sobre las consecuencias socialmente aceptadas del CA, como mecanismo de afrontamiento y aceptación por parte de los pares. Se identifica que regularmente las creencias o expectativas se aprenden por medio de la socialización familiar ya que existe consenso entre padres, madres e hijos en cuanto a los efectos que el alcohol pueda tener en la conducta de las personas, además de que los padres, amigos y medios de comunicación contribuyen a la adquisición de creencias positivas (Mitchell, 2004). En este sentido, que en el AFC se covariaran los errores de los factores 'Beber alcohol ayuda a superar las penas' y 'Beber alcohol ayuda a ser aceptado por el grupo' tiene sentido, ya que ambas creencias son compartidas por adultos y pares. Esto se observa en las canciones y dichos mexicanos los cuales contribuyen a la adquisición de las creencias grupales. En canciones mexicanas se escuchan elementos de exacerbación del alcohol como una forma de afrontar los problemas, como un elemento importante en la vida de los mexicanos para pasarla bien, para llorar las penas y para animar las fiestas (Mitchell, 2004). Lo anterior indica la trasmisión de las creencias de manera vertical y horizontal por parte de pares y padres (Berry et al., 2011).

En cuanto al factor Placer-Hedonismo, las bebidas alcohólicas tienen un impacto profundo a nivel del sistema nervioso central, haciendo que quien consume experimente sensaciones de sedación, euforia o relajación. En adolescentes el hecho de consumir, está vinculado con la experimentación de estas sensaciones placenteras, que son guiadas por la curiosidad, la presión o por el hecho de vivir algo nuevo (Patrick et al., 2010; Shell et al., 2010), además de que en México el CA es un fenómeno con una alta incidencia de casos, cerca del $71 \%$ de la población consume alcohol (Bustos-Gamiño et al., 2019; Villatoro-Velázquez et al., 2017), es probable que este modelo de placer y disfrute esté siendo reproducido por los adolescentes (Higareda et al., 2018) al mirar las actividades que los adultos realizan y lo que simboliza el alcohol en las fiestas, los jaripeos, las reuniones, lo que es llamado cultura subjetiva y objetiva, la primera en forma de normas, creencias y valores asociados con el alcohol y la segunda en forma de los elementos objetivos como las bebidas y los lugares para llevar a cabo esta actividad (Higareda et al., 2018).

El análisis de invarianza por grupos mostró que la escala puede ser utilizada en ambos sexos, ya que los índices del modelo son adecuados en caso de usarse de manera independiente. Es relevante porque no se identificaron estudios con este análisis.

\section{Limitaciones}

Se recomienda que para futuras investigaciones se utilicen muestreos probabilísticos que coadyuven a evitar sesgos por sexo, nivel 
socioeconómico, edad, etc., debido a que se trabajó únicamente con adolescentes escolarizados, por tanto, se sugiere incluir muestras de adolescentes fuera del sistema educativo para ampliar la representatividad. Finalmente, se propone continuar con la línea de investigación para poder obtener la relación con otras escalas que midan las creencias o expectativas para tener indicadores de validez convergente y así fortalecer los índices psicométricos y de validez de constructo de la escala.

\section{Conclusiones}

Por medio de tres pilotos se obtuvo una escala válida y confiable que evalúa las creencias sobre el CA de adolescentes escolarizados mexicanos cuyas edades fluctuaron entre los 11 y 16 años, lo cual es relevante debido a que en esta etapa de vida las y los adolescentes comienzan a desarrollar sus actitudes sobre iniciar su consumo de alcohol. Conocer estas motivaciones razonadas permitirá diseñar intervenciones específicas para incidir en esta problemática de salud pública, además de relacionar estas creencias con otras escalas que contribuyan al desarrollo teórico de la TAP y el CA. La escala fue creada desde un piloto cualitativo, lo cual es valioso debido a que otras medidas carecen de este tipo de análisis previo lo cual coadyuva al desarrollo de instrumentos desde la exploración del fenómeno directamente en el contexto de las personas. Utilizar esta escala permitiría analizar la toma de decisiones razonada sobre el CA en adolescentes, conocer sus motivaciones es importante para realizar campañas de prevención sobre el CA, además de que su aplicación puede ser indiferenciada por sexo debido a que la estructura es adecuada en el grupo de hombres y mujeres adolescentes.

\section{Referencias}

Abad, F. (2011). Medición en ciencias sociales y de la salud. Síntesis.

Ahumada-Cortez, J., Gamez-Medina, M., Valdez, C., \& Moreno, D. (2017). Bullying and legal and illegal drug use in adolescents: A systematic review. Nursing \& Care Open Access Journal, 3(4). https://doi.org/10.15406/nc oaj.2017.03.00082

Ajzen, I. (2002). Residual effects of past on later behavior: Habituation and reasoned action perspectives. Personality and Social Psychology Review, 6(2), 107-122. https://doi.org/10.1207/S15327957PSPR0602_02

Ajzen, I., \& Driver, B. L. (1992). Application of the Theory of Planned Behavior to leisure choice. Journal of Leisure Research, 24(3), 207-224. https://do i.org/10.1080/00222216.1992.11969889

Berruecos, L. (2013). El consumo de alcohol y el alcoholismo en México: el caso de las comunidades indígenas. El Cotidiano, 181, 73-80.

Berry, J. W. (1992). Acculturation and adaptation in a new society. International Migration, 30, 69-85. https://doi.org/10.1111/j.1468-243 5.1992.tb00776.x 
Berry, J. W., Poortinga, Y. H., Breugelmans, S. M., Chasiotis, A., \& Sam, D. L. (2011). Cross-cultural psychology. Cambridge University Press. https://do i.org/10.1017/cbo9780511974274

Brown, T. (2006). Confirmatory factor analysis for applied research. The Guilford Press.

Bustos-Gamiño, M., Reséndiz, E., Villatoro-Velázquez, J. A., \& SotoHernándezz, I. (2019). Problemas Asociados al Consumo de Sustancias Psicoactivas. INPRFM.

Byrne, B. (2012). Handbook of structural equation modeling. The Guilford Press.

Castaño Pérez, A., García del Castillo, J. A., \& Marzo Campos, J. C. (2014). Alcohol consumption and intervening factors observed in university students. Revista Cubana de Salud Pública, 40(1), 1-13.

Chan, G. C. K., Kelly, A. B., Carroll, A., \& Williams, J. W. (2017). Peer drug use and adolescent polysubstance use: Do parenting and school factors moderate this association? Addictive Behaviors, 64, 78-81. https://doi.or g/10.1016/j.addbeh.2016.08.004

Choi, J., Kim, S., Chen, J., \& Dannels, S. (2011). A comparison of maximum likelihood and bayesian estimation for polychoric correlation using Monte Carlo simulation. Journal of Educational and Behavioral Statistics, 36(4), 523-549. https://doi.org/10.3102/1076998610381398

CIJ. (2020). Centros de Integración Juvenil. https://www.gob.mx/salud/cij/es/a rticulos/cij-atendio-a-casi-2-millones-y-medio-de-personas-durante-el-pr imer-trimestre-del-ano?idiom=es

Cortés, M. T., Espejo, B., Giménez, J. A., Luque, L., Gómez, R., \& Motos, P. (2011). Creencias asociadas al consumo intensivo de alcohol entre adolescentes. Saludy Drogas, 11(2), 179-202.

Donovan, J. E., Molina, B. S. G., \& Kelly, T. M. (2009). Alcohol outcome expectancies as socially shared and socialized beliefs. Psychology of Addictive Behaviors, 23(2), 248-259. https://doi.org/10.1037/a0015061

Ferrando, P. J., \& Lorenzo-Seva, U. (2007). A measurement model for Likert responses that incorporates response time. Multivariate Behavioral Research, 42(4), 675-706. https://doi.org/10.1080/0027317070171024 7

Ferrando-Piera, P., \& Anguiano-Carrasco, C. (2010). El análisis factorial como técnica de investigación en psicología. Papeles Del Psicólogo, 31(1), 18-33.

Gerrard, M., Gibbons, F. X., Benthin, A. C., \& Hessling, R. M. (1996). A longitudinal study of the reciprocal nature of risk behaviors and cognitions in adolescents: What you do shapes what you think, and viceversa. Health Psychology, 15(5), 344-354. https://doi.org/10.1037/0278-6133.15.5.34 4

Goldman, M. S., del Boca, F. K., \& Darkes, J. (1999). Alcohol expectancy theory: The application of cognitive neuroscience. En K. E. Leonard \& H. T. Blane (Eds.), The Guilford substance abuse series. Psychological theories of drinking and alcoholism (pp. 203-246). The Guilford Press.

Goldman, M. S., Reich, R. R., \& Darkes, J. (2006). Expectancy as a unifying construct in alcohol-related cognition. En R. W. Wiers \& A. W. Stacy (Eds.), Handbook of implicit cognition and addiction (pp. 105-119). SAGE Publications. https://doi.org/10.4135/9781412976237.n8 
Golpe, S., Isorna, M., Barreiro, C., Braña, T., \& Rial, A. (2017). Consumo intensivo de alcohol en adolescentes: prevalencia, conductas de riesgo y variables asociadas. Adicciones, 29(4), 256-267. https://doi.org/10.2088 2/adicciones.932

Guzmán-Facundo, F., García, B., Rodríguez, L., \& Alonso, M. (2018). Actitud, norma subjetiva y control conductual como predictores del consumo de drogas en jóvenes de zona marginal del norte de México. Frontera Norte, 26(51), 21-44. https://doi.org/10.2307/j.ctv26d9ch.5

Hammer, J. H., Parent, M. C., Spiker, D. A., \& World Health Organization. (2018). Global status report on alcohol and health 2018. WHO. https://d oi.org/10.1037/cou0000248

Hernández Chávez, E. C., Salazar Garza, M. L., Vacio Muro, Ma. D. los A., \& Rodríguez-Kuri, S. E. (2017). Construcción del cuestionario de intención hacia el alcohol para niños mexicanos desde la Teoría de la Conducta Planeada. Universitas Psychologica, 16(2), 1-10. https://doi.org/10.1114 4/javeriana.upsy16-2.iein

Higareda, J., Rivera, S., Romero, A., Reidl, L., \& Flores, M. (2018). Normas y creencias asociadas al consumo de alcohol en adolescentes de Hidalgo: un estudio exploratorio. En S. Rivera, L. Cruz, F. Méndez, P. Velazco, C. Jaén, \& G. Villanueva (Eds.), La psicología social en México XVII (pp. 407-428). AMEPSO.

Hodder, R. K., Campbell, E., Gilligan, C., Lee, H., Lecathelinais, C., Green, S., MacDonald, M., \& Wiggers, J. (2018). Association between Australian adolescent alcohol use and alcohol use risk and protective factors in 2011 and 2014. Drug and Alcohol Review, 37, S22-S33. https://doi.org/10.11 $11 /$ dar. 12623

Inter-American Observatory on Drugs. (2019). Report on Drug Use in the Americas 2019, Executive Summary. https://www.issup.net/knowledge-share/publications/2018-1 $1 /$ report-drug-use-americas-2019-executive-summary

Jones, B. T., Corbin, W., \& Fromme, K. (2001). A review of expectancy theory and alcohol consumption. Addiction, 96(1), 57-72). https://doi.org/10. 1046/j.1360-0443.2001.961575.x

Jones, S. (2016). Parental provision of alcohol: A TPB-framed review of the literature. Health Promotion International, 31(3), 562-571. https://doi. org/doi.org/10.1093/heapro/dav028

Krippendorff, K. (2018). Content analysis. SAGE Publications.

López-Cisneros, M. A., Villar-Luis, M. A., Alonso-Castillo, M. M., AlonsoCastillo, M. T. de J., \& Aguilar, L. R. (2013). Attitudes towards consumption and non-consumption of alcohol among high school students in México. Revista Da Escola de Enfermagem, 47(4), 815-821. h ttps://doi.org/10.1590/S0080-623420130000400007

Maggs, J. L., Staff, J., Patrick, M. E., Wray-Lake, L., \& Schulenberg, J. E. (2015). Alcohol use at the cusp of adolescence: A prospective national birth cohort study of prevalence and risk factors. Journal of Adolescent Health, 56(6), 639-645. https://doi.org/10.1016/j.jadohealth.2015.02.010

Martínez, C. M., \& Sepúlveda, M. A. R. (2012). Introducción al análisis factorial exploratorio. Revista Colombiana de Psiquiatría, 41(1), 197-207. https:/ /doi.org/10.1016/s0034-7450(14)60077-9 
Martínez-Cardona, M., Muñoz-Borja, P., \& Barberena-Borja, N. (2019). Alcohol use and beliefs among adolescents and young people. Archivos Venezolanos de Farmacología y Terapéutica, 39(4), 487-492.

Matsumoto, D. (2001). The handbook of culture and psychology. Oxford University Press.

Mitchell, T. (2004).Intoxicated identities: Alcohol'spower in Mexican history and culture. Routledge. https://doi.org/10.4324/9780203504567

Montaño, D. E., \& Kasprzyk, D. (2015). Theory of reasoned action, theory of planned behavior, and the integrated behavioral model. En K. Glanz, B. Rimer, \& K. Viswanath (Eds.), Health behavior: Theory, research and practice (pp. 95-110). Wiley.

Musitu Ochoa, G. (2014).Why do adolescents have a low risk perception on alcohol use? The experts view. Revista Metamorfosis: Revista del Centro Reina Sofia sobre Adolescencia y Juventud 1, 55-73.

Nunnally, J., \& Bernstein, I. (1995). Psychometric theory. Mc Graw-Hill.

Oliva-Delgado, A., Parra-Jiménez, A., \& Sánchez-Queija, I. (2008). Consumo de sustancias durante la adolescencia: trayectorias evolutivas y consecuencias para el ajuste psicológico. International Journal of Clinical and Health Psychology, 153-169.

Organización Panamericana de la Salud. (2015). Informe sobre la situación regional sobre el alcohol y la salud en las Américas. OPS.

Patrick, M., Wray-Lake, A., Finlay, A., \& Maggs, J. (2010). Long arm of expectancies: Adolescent alcohol expectancies predict adult alcohol use. Alcohol and Alcoholism, 45(1), 17-24. https://doi.org/10.1093/alcalc/ag p066

Pechorro, P., Oliveira, J. P., Gonçalves, R. A., \& Jesus, S. N. (2018). Psychometric properties of a short version of the Barratt impulsiveness scale - 11 among a school sample of Portuguese adolescents. Revista Iberoamericana de Diagnóstico y Evaluacion Psicologica, 2(47), 157-170. https://doi.org/10 .21865/RIDEP47.2.11

Peltzer, R. I., Conde, K., Biscarra, M. A., Lichtenberger, A., \& Cremonte, M. (2017). Broadening the evidence for the theory of planned behavior: Predicting heavy episodic drinking in Argentinean female and male youth. Health and Addictions/Saludy Drogas, 17(2), 159-167. https://doi.org/ 10.21134/haaj.v17i2.323

Pons, J., \& Buelga, S. (2011). Factores asociados al consumo juvenil de alcohol: una revisión desde una perspectiva psicosocial y ecológica. Psychosocial Intervention, 20(1), 75-94. https://doi.org/10.5093/in2011v20n1a7

Randolph, K. A., Archuleta, A., Smith, T., \& Teasley, M. (2013). Beliefs about alcohol use among youths during early adolescence. Journal of Child and Adolescent Substance Abuse, 22(4), 295-320. https://doi.org/10.1080/10 67828X.2012.747893

Reyes-Lagunes, I., \& García y Barragán, L. (2008). Procedimiento de validación psicométrica culturalmente relevante: un ejemplo. En A. S., Rivera, R., Díaz-Loving, A. R. Sánchez, \& Reyes-Lagunes I. (Eds.), La psicología social en México XII: Vol. XII (pp. 1-7). AMEPSO.

Ringwalt, C. L., Paschall, M. J., Grube, J. W., Miller, T. R., Warren, K. R., Gidi, V., Fisher, D. A., \& Goldberg, A. (2020). Alcohol availability, use, and harms among adolescents in three Mexican cities. Journal of Drug 
Education, 49(1-2), 55-68. https://doi.org/10.1177/00472379209293 31

Rodriguez, M. A. F., Moreno, S. D., \& Gomez, Y. F. (2019). The influence of gender roles in alcohol consumption: A qualitative study of adolescents and young adults in Asturias/La influencia de los roles de género en el consumo de alcohol: estudio cualitativo en adolescentes y jovenes en Asturias. Adicciones, 31(4), 260-274.

Secretaría de Salud. (2015). Encuesta Nacional de Consumo de Drogas en Estudiantes 2014 (ENCODE). Secretaría de Salud, México.

Settles, R. E., Zapolski, T. C. B., \& Smith, G. T. (2014). Longitudinal test of a developmental model of the transition to early drinking. Journal of Abnormal Psychology, 123(1), 141-151. https://doi.org/10.1037/a0035 670

Shell, D. F., Newman, I. M., \& Xiaoyi, F. (2010). The influence of cultural orientation, alcohol expectancies and self-efficacy on adolescent drinking behavior in Beijing. Addiction, 105(9), 1608-1615. https://doi.org/10.1 $111 /$ j.1360-0443.2010.03006.x

Spear, L. P. (2002). The adolescent brain and the college drinker: Biological basis of propensity to use and misuse alcohol.Journal of Studies on Alcohol, 63(14), 71-81. https://doi.org/10.15288/jsas.2002.s14.71

Tabachnick, B., \& Fidell, L. (2007). Using multivariate statistics (5th Edition). Allyn \& Bacon/Pearson Education.

Tavares, D. O. M. L., dos Santos Reinaldo, A. M., Villa, E. A., Pereira, M. O., \& Monteiro, M. A. M. (2019). Informação, crenças e atitudes de escolares acerca do uso de Álcool e outras Drogas. SMAD Revista Eletrônica Saúde Mental Álcool e Drogas (Edição Em Português), 15(2), 45-51. https://doi .org/10.11606/issn.1806-6976.smad.2019.000408

Templos-Núñez, L., Villalobos-Gallegos, L., Cervera-Ballesteros, J., \& MarínNavarrete, R. (2013). Alcohol outcome expectancies questionnaire (CERCA): Psychometric properties in inpatients for addictions in Mexico. Adicciones, 25(4), 327-332. https://doi.org/10.20882/adiccion es.34

Timmerman, M. E., \& Lorenzo-Seva, U. (2011). Dimensionality assessment of ordered polytomous items with parallel analysis. Psychological Methods, 16(2), 209-220. https://doi.org/10.1037/a0023353

UNESCO, I. (2013). Clasificación Internacional Normalizada de la Educación. CINE 2011.

Villatoro-Velázquez, J. A., Reséndiz, E. E., Mujica, S. A., BretónCirret, M., Cañas-Martínez, V., Soto-Hernández, I., Fregosso-Ito, D., Fleiz-Bautista, C., Medina-Mora, M., Gutiérrez, J., FrancoNuñez, A., Romero, M., \& Mendoza, L. (2017). Encuesta Nacional de Consumo de Drogas, Alcohol y Tabaco. Secretaría de Salud. https://www.gob.mx/salud\%7Cconadic/acciones-y-programas/e ncuesta-nacional-de-consumo-de-drogas-alcohol-y-tabaco-encodat-2016 $-2017-136758$

WHO. (2013). Global Student Health Survey (GSHS). World Health Organization, Department of Chronic Diseases and Health Promotion, January, 1-16. http://www.who.int/chp/gshs/methodology/en/index.h tml\%5Cnhttp://www.cdc.gov/gshs/questionnaire/index.htm 
Wilsnack, R. W., Wilsnack, S. C., Gmel, G., \& Kantor, L. (2018). Gender differences in binge drinking: Prevalence, predictors, and consequences. Alcohol Research: Current Reviews, 39(1), 57-76.

Woolfolk, A. (2010). Educational psychology in teacher education. Educational Psychologist, 35(4), 257-270. https://doi.org/10.1207/S15326985EP35 04_04

Ximénez-Gómez, C., \& García, A. (2005). Comparación de los métodos de estimación de máxima verosimilitud y mínimos cuadrados no ponderados en el análisis factorial confirmatorio mediante simulación Monte Carlo. Psicothema, 17(3), 528-535. 